\title{
Severe inflammatory arthritis and lymphadenopathy in the absence of TNF
}

\author{
Ian K. Campbell, Kristy O’Donnell, Kate E. Lawlor, and Ian P. Wicks
}

Reid Rheumatology Laboratory, Division of Autoimmunity and Transplantation, Walter and Eliza Hall Institute of Medical Research, Parkville, Victoria, Australia

Address correspondence to: Ian P. Wicks, Reid Rheumatology Laboratory, Division of Autoimmunity and Transplantation, Walter and Eliza Hall Institute of Medical Research, Post Office Royal Melbourne Hospital, Victoria 3050, Australia.

Phone: 61-3-9345-2466; Fax: 61-3-9347-0852; E-mail: wicks@wehi.edu.au.

Received for publication March 13, 2001, and accepted in revised form May 14, 2001.

It has been postulated that TNF has a pivotal role in a cytokine cascade that results in joint inflammation and destruction in rheumatoid arthritis (RA). To evaluate this, we examined the response of TNF-deficient $\left(\mathrm{Tnf}^{/-}\right)$mice in two models of RA. Collagen-induced arthritis (CIA) was induced by injection of chick type II collagen (CII) in CFA. Tnf $/-$ mice had some reduction in the clinical parameters of CIA and, on histology, significantly more normal joints. However, severe disease was evident in $54 \%$ of arthritic $\mathrm{Tnf}^{/-}$joints. Tnf ${ }^{/-}$mice had impaired Ig class switching, but preserved $\mathrm{T}$ cell proliferative responses to CII and enhanced IFN- $\gamma$ production. Interestingly, CII-immunized $\mathrm{Tnf}^{/-}$mice developed lymphadenopathy and splenomegaly associated with increased memory $\mathrm{CD}^{+}$ $\mathrm{T}$ cells and activated lymph node B cells. Acute inflammatory arthritis was also reduced in $\mathrm{Tnf} / \mathrm{-}$ mice, although again some mice exhibited severe disease. We conclude that TNF is important but not essential for inflammatory arthritis; in each model, severe arthritis could proceed even in the complete absence of TNF. These results call into doubt the concept that TNF is obligatory for chronic autoimmune and acute inflammatory arthritis and provide a rationale for further studies into TNF-independent cytokine pathways in arthritis.

J. Clin. Invest. 107:1519-1527 (2001).

\section{Introduction}

The cytokine TNF has a remarkable range of actions in inflammation, infection, and immunity (1). Rheumatoid arthritis (RA) is associated with local overproduction of TNF, and inhibition of TNF ameliorates experimental arthritis. These data have given rise to a new model for the pathogenesis of RA, which postulates that TNF occupies a pivotal position in a cytokine cascade that regulates the production of inflammatory mediators such as $\operatorname{IL}-1(2,3)$. This hypothesis has major implications for understanding RA and also for how it should be treated. However, the absolute dependence of inflammatory arthritis on TNF has never been directly examined.

Collagen-induced arthritis (CIA) is a chronic autoimmune model of human RA that is widely used for dissecting molecular and cellular mediators of this disease, as well as for evaluating possible therapeutic agents. A limitation of this model for the study of transgenic and knockout mice is an apparent MHC class II restriction to the $\mathrm{q}$ and $\mathrm{r}$ haplotypes (4). Since knockout mice are usually generated on the 129 or C57BL/6 (B6) genetic backgrounds, both of which bear $\mathrm{H}-2^{\mathrm{b}}$, it has been necessary to backcross knockout mice onto the susceptible DBA/1 (H-29) strain for studies in CIA (5-11). However, the close proximity of the $\operatorname{Tnf}$ gene to the $\mathrm{H}-2$ locus and immune response genes on chromosome 17 prohibits this approach. We reported recently a new immunization procedure whereby the CIA model can be applied directly to $\mathrm{H}-2^{\mathrm{b}}$ mice (12), thereby circumventing the need for backcross of knockout mice onto the DBA/ 1 strain.

In keeping with previous roles proposed for TNF, TNF-deficient ( $\left.\operatorname{Tn} f^{\prime-}\right)$ mice succumbed more readily to challenge with infectious agents and were resistant to endotoxic shock following sensitization with D-galactosamine (13-15). Tnf ${ }^{\prime-}$ mice had normal peripheral blood leukocyte subsets and normal in vitro function of neutrophils, macrophages, $\mathrm{CD}^{+}$cells, and $\mathrm{CD}^{+} \mathrm{T}$ cells (14). Interestingly, following intraperitoneal injection with LPS $(13,14)$, there was normal production of cytokines in Tnf ${ }^{\prime-}$ mice, including IL-1 $\beta$. Tnf ${ }^{\prime-}$ mice developed normally apart from absence of Peyer's patches and abnormal microarchitectural lymphoid structure in secondary lymphoid tissues. Although the $\mathrm{T}$ and $\mathrm{B}$ cell number was not affected, $T$ and $B$ cell regions in lymphoid follicles were disorganized and primary B cell follicles and germinal centers were absent. Ig class switching for $\mathrm{T}$ cell-dependent antigens was impaired in 129 Tnf/- mice (13, 14), but not B6 Tnf/- mice (15).

In this study, we employed B6 Tnf/- mice to examine the absolute dependence of inflammatory arthritis on TNF. Two models were examined: CIA and methylated BSA/IL-1-induced (mBSA/IL-1-induced) arthritis, an 
acute monoarticular arthritis. Each of these models has features of human RA. We show that TNF is important but is not absolutely required for either CIA or mBSA/IL-1-induced acute inflammatory arthritis. In each model there was overall reduced disease in the absence of TNF, but severe arthritis still developed in some mice. The profile of inflammatory cytokines found in acutely inflamed synovium was similar in Tnf ${ }^{\prime-}$ and wild-type (WT) mice. Unexpectedly, Tnf ${ }^{\prime-}$ mice developed lymphadenopathy and splenomegaly in response to immunization with CII/CFA associated with the accumulation of memory $\mathrm{CD} 4^{+} \mathrm{T}$ cells in both sites and of activated lymph node (LN) B cells. These results question the concept of TNF as an obligatory cytokine in chronic autoimmune and acute inflammatory arthritis, highlight a number of important biological functions for TNF in the adaptive immune response, and may have implications for the increasing clinical use of TNF antagonists in RA.

\section{Methods}

Mice. Mice deficient in the Tnf gene $\left(\operatorname{Tnf} f^{-}\right)$were generated by direct gene targeting of $\mathrm{B} 6$ embryonic stem cells as described (15) and were provided by M. Smyth (Austin Research Institute, Heidelberg, Australia), with permission from J. Sedgwick (Centenary Institute, Sydney, Australia). Tnf $f^{/-}$mice were housed in filter-topped cages at all times. B6 mice, obtained from the Walter and Eliza Hall Institute of Medical Research animal services (Kew, Australia), were used as WT controls in all experiments. All animal procedures were approved by the institutional ethics committee.

CIA. Chick type II collagen (CII; Sigma Chemical Co., St Louis, Missouri, USA), dissolved in $10 \mathrm{mM}$ acetic acid at a concentration of $2 \mathrm{mg} / \mathrm{ml}$, was emulsified in an equal volume of CFA containing $5 \mathrm{mg} / \mathrm{ml}$ heat-killed Mycobacterium tuberculosis (strain H37Ra; Difco Laboratories, Detroit, Michigan, USA), as described previously (12). Arthritis was induced by injecting mice intradermally at several sites into the base of the tail with $100 \mu \mathrm{l}$ emulsion; the process was repeated 21 days later.

Animals were assessed for erythema and swelling of limbs, and a clinical score was allocated to each mouse 2-3 times per week for up to 60 days. The scoring system was as described previously (16) with a maximum score of 12 per mouse. At sacrifice, the paws were removed, fixed, decalcified, and processed for paraffin embedding. Hematoxylin and eosin-stained sections $(5 \mu \mathrm{m})$ of the front and rear paws of four mice from each genotype with the highest clinical scores were evaluated as described previously (16). Clinical and histological evaluations were assessed by two independent investigators blinded to the experimental groups. At sacrifice, blood was taken for determination of serum anti-CII Ab.

Acute monoarticular arthritis. Acute monoarticular arthritis was induced and assessed as described (17). Briefly, mice were injected intra-articularly into the knee joint with $200 \mu \mathrm{g}$ mBSA (Sigma Chemical Co.), and the contralateral joint received the same volume $(10 \mu \mathrm{l})$ of vehicle (normal saline). Recombinant human IL-1 3 (250 ng) was next injected subcutaneously into the footpad and repeated on the subsequent 2 days. Mice were sacrificed 7 days after the initial injections, and the rear limbs were removed and processed, as above, for histological assessment of hematoxylin and eosin-stained frontal sections of the stifle joints. Coded sections were assessed without knowledge of the experimental groups and graded in severity from 0 (normal) to 5 (severe) for five components, including joint exudate, synovitis, pannus formation, cartilage degradation, and bone degradation. Based on the histological scores, joints were classified as demonstrating inflammatory arthritis if there was an exudate score of 1 or more and a synovitis score of 2 or more. Destructive arthritis was defined for joints that scored 2 or more for pannus or 1 or more for either cartilage degradation or bone degradation.

ELISA for detection of Ab's to CII. ELISAs were performed to detect Ab's to CII as described previously (18). Horseradish peroxidase-conjugated goat antimouse IgG (Sigma Chemical Co.) or IgM (Southern Biotechnology Associates, Birmingham, Alabama, USA) antisera were used as detection Ab's. Standard curves (in arbitrary units per milliliter) for anti-CII IgG and IgM were constructed from the pooled sera of CII

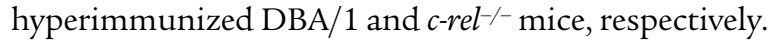

$T$ cell proliferation assay. These were performed as described (16). Briefly, inguinal LNs were harvested and pooled from mice $(n=6) 67$ days after primary immunization with CII (as described above). LN cells were sieved to form a single cell suspension and washed in RPMI media containing 2-mercaptoethanol $(50 \mu \mathrm{M})$ and $5 \%(\mathrm{vol} / \mathrm{vol}) \mathrm{FCS}$. LN cells $\left(4 \times 10^{5}\right)$ in 200 $\mu \mathrm{l}$ media were added to round-bottomed wells in a 96well plate (Becton Dickinson Labware, Franklin Lakes, New Jersey, USA) and incubated at $37^{\circ} \mathrm{C}\left(5 \% \mathrm{CO}_{2}\right)$ for 72 hours with $0-100 \mu \mathrm{g} / \mathrm{ml}$ ( $n=6$ wells per group) of denatured CII (boiled for 10 minutes). After 48 hours, aliquots were removed for measurement of IFN- $\gamma$. For the final 6 hours of incubation, $5 \mu \mathrm{Ci} / \mathrm{ml}\left[{ }^{3} \mathrm{H}\right]$ thymidine (Amersham International, Amersham, United Kingdom) was added and its incorporation used as a measure of $\mathrm{T}$ cell proliferation.

ELISA for IFN- $\gamma$. IFN- $\gamma$ in supernatants from CII-stimulated LN cells was assayed by capture ELISA using paired mAb's (PharMingen, San Diego, California, USA) according to the manufacturer's instructions.

Assay for antinuclear $A b$ 's. Sera (diluted 1:40 in PBS $/ 0.5 \%$ BSA $)$ taken from WT $(n=28)$ and $\mathrm{Tnf}^{-/-}$ $(n=26)$ mice 60-67 days after primary immunization with CII were used for indirect immunofluorescence staining of Hep-2 cells. NOD mouse serum was used as a positive control.

Immunobistochemistry on $L N$ and spleen. Acetone-fixed frozen sections were stained with biotinylated mouse mAb's to B220 (clone RA3.6B2; American Type Culture Collection, Manassas, Virginia, USA) or CD4 (clone GK1.5, gift from T. Mandel, Walter and Eliza Hall Institute of Medical Research). Staining was visu- 

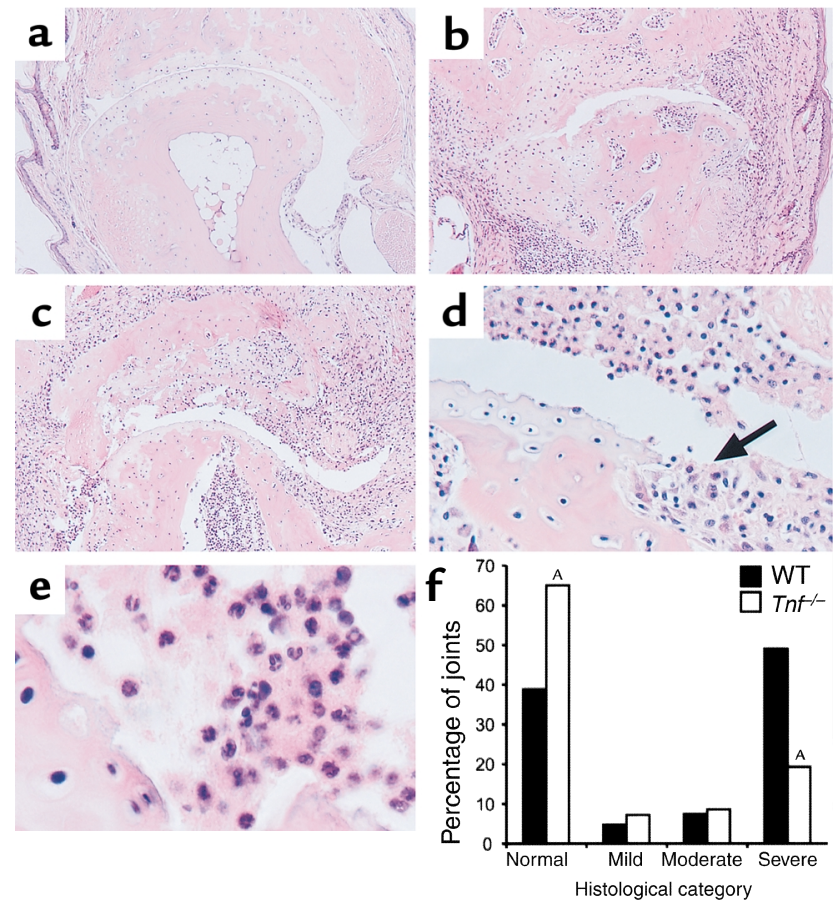

Figure 1

Histopathological assessment of joints from Cll-immunized Tnf $/-$ and WT mice. At 60 days after primary immunization with CII, mice were sacrificed, their limbs removed, and the paws processed for histological assessment as described in Methods. Frontal sections of normal (a) and severely arthritic interphalangeal joints of WT (b) and $\mathrm{Tnf}^{\prime-}$ (c) mice are shown. (d, e) Higher magnification views of some of the histopathological features of $\mathbf{c}$. Hematoxylin and eosin stained sections, original magnification: $(\mathbf{a}-\mathbf{c}) \times 100,(\mathbf{d}) \times 400$, and (e) $\times 1,000$. Arrow indicates invasive synovial pannus. (f) Sections were blindly graded as either normal, mild, moderate, or severe. Results show the percentage of joints from $\operatorname{Tnf}^{-/}(n=140$ joints $)$ and WT ( $n=147$ joints) mice in each group. ${ }^{A} P<0.001$, compared with the same histological grade in WT mice.

alized using the VectaStain Elite ABC kit (Vector Laboratories, Burlingame, California, USA), according to the manufacturer's instructions, with diamino-benzidine as the substrate.

FACS analysis. Inguinal LN and spleen cells were sieved to create single cell suspensions and washed in RPMI containing 10\% (vol/vol) FCS. Erythrocytes were removed from spleen cell suspensions using red cell removal buffer $\left(0.16 \mathrm{M} \mathrm{NH}_{4} \mathrm{Cl}, 0.13 \mathrm{mM}\right.$ EDTA, $12 \mathrm{mM} \mathrm{NaHCO} 3$ ). Cells were stained with $\mathrm{Ab}$ 's to the following molecules: biotinylated anti-B220 or CD62L (clone MEL-14; PharMingen), followed by streptavidin-phycoerythrin (streptavidin-PE) or Tricolor (Caltag Laboratories Inc., Burlingame, California, USA); PE-conjugated mAb's to CD4 (clone CTCD4; Caltag Laboratories Inc.), CD8 (clone 53-6.7; Sigma Chemical Co.), CD25 (clone PC61 5.3; Caltag Laboratories Inc.) and CD11b (clone M1/70.15; Caltag Laboratories Inc.), and FITC-conjugated Ab's to CD4 (H129.19; Sigma Chemical Co.), IgM (polyclonal; Southern Biotechnology Associates), neutrophil marker (clone 7/4; Caltag Laboratories Inc.), CD44 (clone IM7; PharMingen), and I-A $\mathrm{A}^{\mathrm{b}}$ (clone M5/114; American Type Culture Collection). The level of nonspecific staining was determined using isotypematched Ab's of irrelevant specificity, and propidium iodide staining was used to assess cell viability (Calbiochem-Novabiochem, La Jolla, California, USA). Cells were analyzed by flow cytometry on a FACScan using Cell Quest software (Becton Dickinson, San Jose, California, USA).

$R$ Nase protection analysis of cytokine expression. Acute monoarticular arthritis was induced in mice by injection with mBSA and IL-1 $\beta$. On day 7 , synovial tissue was dissected from the knee joints, snap-frozen, and ground into a powder using a mortar and pestle. Muscle adjacent to the synovium was similarly treated and used as a negative control. Total RNA was extracted using RNAzol B reagent (Tel-Test Inc., Friendswood, Texas, USA) and analyzed for cytokine expression using the RiboQuant RNase Protection System (PharMingen) according to the manufacturer's instructions. The murine cytokine template set $\mathrm{mCK} 2 \mathrm{~b}$ (PharMingen) was used to obtain radiolabeled antisense RNA probes for IL-1 $\alpha$, IL-1 $\beta$, IL-1Ra, IL-18, IL-6, IFN- $\gamma$, and macrophage migration inhibitory factor (MIF). The mCK3 template set (PharMingen) was used to amplify TNF- $\alpha$. The housekeeping gene L32 was used as a control for RNA loading in both template sets. Dried polyacrylamide gels were visualized (after an overnight exposure) with a PhosphorImager (Molecular Dynamics, Sunnyvale, California, USA), using Image Quant software.

Statistics. For CIA clinical scores and mBSA/IL-1 acute arthritis histological scores the Mann-Whitney twosample rank test was used to determine the level of significance between means of groups. For data pertaining to serum $\mathrm{Ab}$, spleen and $\mathrm{LN}$ weights, and cell numbers, Student's $t$ test for the difference of two means was employed. The incidence of arthritis between different groups and the histological assessments of CIA were evaluated by the $\chi^{2}$ test. For each test $P$ values less than 0.05 were considered statistically significant.

\section{Results}

Tnf/- mice can develop severe CIA. To examine the requirement for TNF in a model of chronic autoimmune arthritis, B6 WT mice and B6 mice homozygous for a null mutation in the Tnf gene were compared for incidence and severity of CIA induced by intradermal immunization with CII in CFA, followed by a boost injection 21 days later. We showed previously that this procedure reproducibly elicits arthritis in B6 mice and is dependent on T and B cells (12). Paws were serially assessed and a clinical score given for each mouse according to the procedure outlined in Methods. Tnf $f^{-1}$ mice developed CIA with a lower incidence and severity and a later time of onset than WT mice, although none of these differences was statistically significant (Table 1). The clinical scores of the arthritic $\operatorname{Tnf}^{\prime /-}$ mice ranged from 1-12 (median 3) 

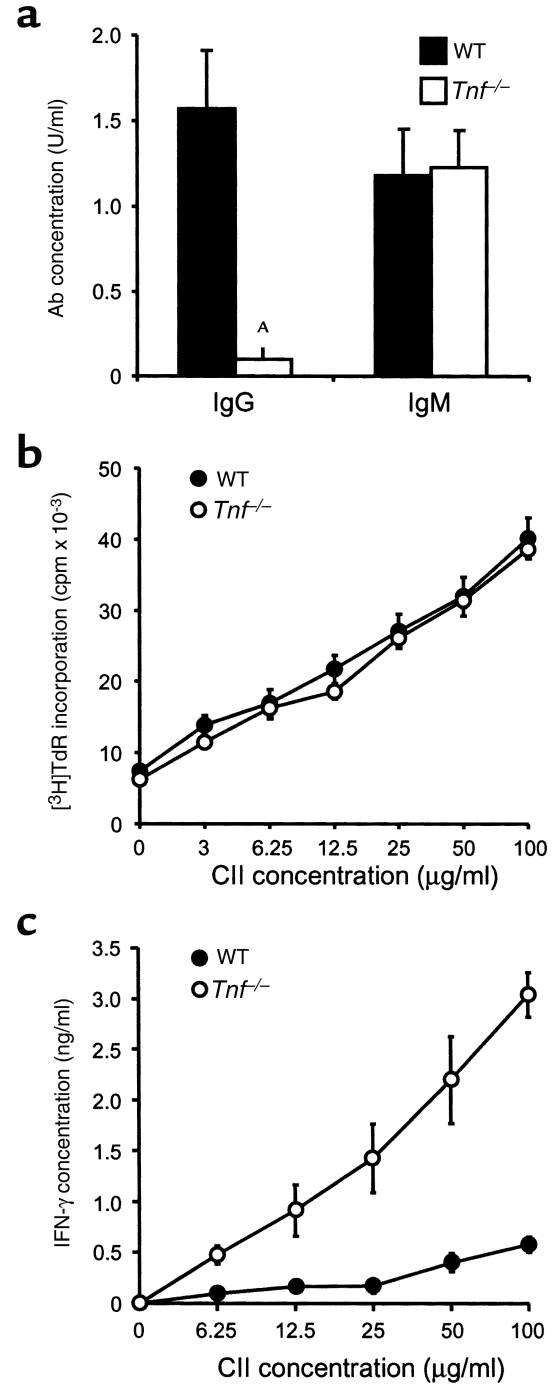

Figure 2

Humoral and cellular responses to $\mathrm{CII}$ in $\mathrm{Tnf}^{-1-}$ and WT mice. (a) Circulating levels of CII-specific Ab's (IgG and IgM) were determined in individual sera from $\operatorname{Tnf}^{\prime-}(n=14)$ and WT $(n=16)$ mice at 60 days after primary immunization with CII. Results show the mean plus SEM values for $\lg G$ and $\lg M$ in arbitrary units per milliliter. ${ }^{A} P<0.001$ compared with WT. (b) T cell proliferation and (c) IFN- $\gamma$ production in response to $\mathrm{CII}_{\text {in }} \mathrm{Tnf}^{\prime-}$ and WT mice. Single cell suspensions of inguinal LN cells were prepared from mice $(\mathbf{b}: n=6$; $\mathbf{c}$ : $n=3) 67$ days after primary immunization with CII. For $\mathbf{b}$, cells were stimulated for 72 hours with different doses of denatured $\mathrm{CII}$ and labeled with $\left[{ }^{3} \mathrm{H}\right]$ thymidine $(\mathrm{TdR})$ over the last 6 hours of culture to measure antigen-specific $T$ cell proliferation. There was no difference between the proliferative responses to $\mathrm{CII}$ of the two groups at any dose $(P>0.05)$. For $\mathbf{c}$, IFN- $\gamma$ levels were determined by ELISA on culture supernatants removed at 48 hours. The $\mathrm{LN}$ cells of $\mathrm{Tnf}^{-1}$ - mice produced significantly higher IFN- $\gamma$ levels than those of WT mice $(P<0.001$; two-way ANOVA $)$. For $\mathbf{b}$ and $\mathbf{c}$, means \pm SEM are shown.

compared with 1-12 (median 4.5) in WT mice; where 1 represents slight swelling in a single paw and 12 is severe swelling and ankylosis in all four paws. TNFdeficient mice could therefore develop severe CIA comparable to WT controls.
Histological analysis of CIA in Tnf/- mice. To further evaluate arthritis, hematoxylin and eosin-stained sections of paws from $\mathrm{Tnf}^{\prime-}$ and WT mice with the highest clinical scores were assessed by histology. Overall, for the $\mathrm{Tnf}^{/ /}$- mice there was a significantly greater percentage of joints in the normal category and a significantly lower percentage of joints in the severe category, compared with the WT mice (Figure 1f, $P<0.001$ for each), collectively indicating reduced disease. However, $20 \%$ of the joints examined from these $\mathrm{Tnf}^{/-}$mice (i.e., $54 \%$ of arthritic joints) showed severe pathology (Figure 1f) with similar histological features to the most markedly affected WT mouse joints (compare Figure 1, $\mathrm{c}$ and $\mathrm{b}$, respectively). These included the characteristic features of inflammatory cell infiltration (Figure 1e) and invasive pannus tissue formation associated with bone and cartilage degradation (Figure 1d, arrow). These data suggest that TNF plays an important but not indispensable role in CIA and that once disease is initiated, it can proceed fully.

Altered bumoral and cellular immune responses to CII in Tnf ${ }^{\prime-}$ mice. Both humoral and cellular immune responses to CII are necessary for full development of CIA $(12,19)$, so these were investigated in the Tnf/and WT mice. Tnf $/-$ mice had a significantly reduced IgG response, but a normal IgM response, to CII compared with the WT controls (Figure 2a). Some RA patients treated with TNF antagonists develop new antinuclear Ab's (ANAs). These were not found in any of the Tnf ${ }^{\prime-}$ or WT mice (data not shown). To assess the cellular immune response to CII, in vitro $\mathrm{T}$ cell proliferation and IFN- $\gamma$ production were compared for $\mathrm{Tnf}^{/-}$and WT mice. Inguinal LN cells were prepared from mice following immunization with CII and stimulated in vitro with $0-100 \mu \mathrm{g} / \mathrm{ml}$ of denatured CII. Both Tnf ${ }^{\prime-}$ and WT mouse LN cells proliferated to a similar degree in a dose-dependent manner, demonstrating antigen-specific $\mathrm{T}$ cell responses (Figure $2 \mathrm{~b}$ ). In contrast, IFN- $\gamma$ production was markedly increased in the LN cells of Tnf $/-$ mice compared with WT mice (Figure 2c).

Lymphadenopathy and splenomegaly in Tnf/- mice following immunization with CII. At the time of sacrifice (60-67 days after primary immunization with CII) Tnf/- mice had markedly enlarged inguinal LNs and spleens compared with similarly treated WT mice (Figure 3, a and b). Such differences were not apparent in nonimmunized mice (not shown). There was a highly significant increase $(P<0.0001)$ in the weights of inguinal LNs and spleens from immunized $\mathrm{Tnf}^{/} /$- mice compared with immunized WT mice, but no such difference in naive animals (Figure $3, \mathrm{c}$ and d). The most striking difference was in the inguinal LNs where CII-immunized $\mathrm{Tnf} /-$ mice had an almost 50-fold increase in weight over naive mice (101.8 $\pm 7.2 \mathrm{mg}$ versus $2.2 \pm 0.2 \mathrm{mg}$, respectively; mean \pm SEM) compared with a 13-fold increase for WT mice $(28.6 \pm 2.5$ $\mathrm{mg}$ ). While lymphadenopathy was a uniform feature of immunized Tnf/- mice, there was no correlation between the weights of lymphoid organs and whether the CII- 

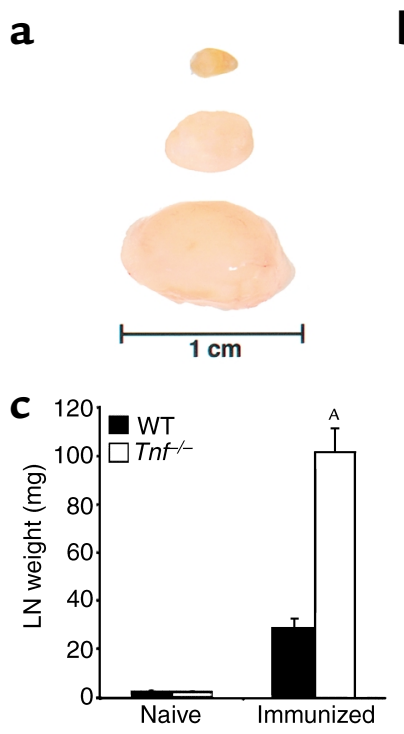

b
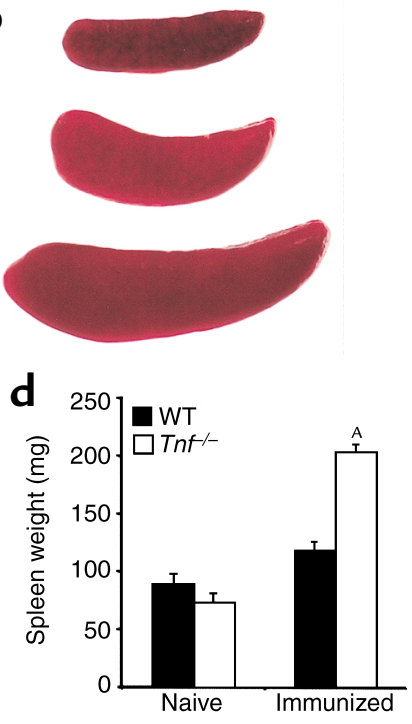

immunized mice developed CIA (data not shown). Immunohistological analyses of the enlarged inguinal LNs and spleens of CII-immunized Tnf $/$ - mice confirmed previous findings (13-15) of aberrant microarchitecture in $\mathrm{T}$ and $\mathrm{B}$ cell lymphoid regions (not shown). Increased $\mathrm{CD}^{+}$memory $T$ cells in the lymphoid organs of CII-immunized $\mathrm{Tnf}^{\prime-}$ mice. FACS analyses of single cell suspensions of LN and spleen were performed to examine leukocyte subsets in CII-immunized mice. The numbers of each subset of leukocytes were equivalent in naive Tnf ${ }^{--}$and WT mice (Figure 4). Following immunization with CII, an expansion of all cell types was observed in the inguinal LN of Tnf ${ }^{/-}$mice compared with WT mice. The number of B cells was increased in the LNs of immunized Tnf ${ }^{\prime-}$ mice, but not in the spleen (Figure 4). LN B cells in Tnf/- mice had greater forward scatter than WT B cells, consistent with activation (not shown). The number of $\mathrm{CD}^{+} \mathrm{T}$ lymphocytes was increased in both the $\mathrm{LN}$ and spleen of immunized Tnf/- mice (Figure 4). Ab's to CD25, CD44, and CD62L (L-selectin) were used to characterize the $\mathrm{CD}^{+}$subset of $T$ cells into recently activated $\left(\mathrm{CD} 25^{+}\right)$, naive $\left(\mathrm{CD} 44^{\mathrm{lo}}\right.$, CD62L $\left.\mathrm{L}^{\mathrm{hi}}\right)$, or memory $\left(\mathrm{CD} 44^{\mathrm{hi}}\right.$, CD62 $\mathrm{L}^{\mathrm{lo}}$ ) phenotypes (20) (Figure 5a). Only $3 \%$ of the $\mathrm{CD}^{+}$cells expressed the early activation marker, CD25 (IL-2 receptor $\alpha$; data not shown). However, $\mathrm{Tnf}^{-1-}$ mice showed a striking increase in memory-type $\mathrm{CD}^{+} \mathrm{T}$ cells after immunization in both the LN and spleen compared with WT mice (Figure $5 \mathrm{~b}, P<0.05$ and $P<0.01$, respectively), which most likely accounts for the enhanced CII-specific IFN- $\gamma$ production seen in vitro (Figure 2c).

\section{Figure 4}

Cellular composition of the inguinal LN and spleen of Tnf ${ }^{/-}$and $\mathrm{WT}$ mice by FACS analysis. Single cell suspensions were prepared from mice that were uninjected (naive: $n=3$ ) or injected with CII 60 days previously (immunized: $n=7$ ). The cells were counted and phenotyped by FACS staining with the Ab's described in Methods. Results show the mean number $( \pm$ SEM) of each cell type per organ. M $\phi$, macrophage. ${ }^{A} P<0.05 ;{ }^{B} P<0.01 ;{ }^{C} P<0.001$, compared with WT immunized mice.

\section{Figure 3}

Enlarged inguinal $\mathrm{LN}$ and spleen of $\mathrm{TnF}^{\prime-}$ mice immunized with $\mathrm{CII}$ in the presence of CFA. WT and $\mathrm{Tnf}^{-1}$ mice were immunized by injection of CII/CFA emulsion at $\mathrm{d} 0$ and 21, as described in Methods. At day 60 , the animals were sacrificed and their inguinal $L N s$ and spleens were removed, blotted dry, and weighed. Representative LNs (a) and spleens (b) of naive WT (upper), immunized WT (middle), and immunized $\mathrm{Tnf}^{-1}$ (lower) mice are shown. The horizontal bar represents a $1-\mathrm{cm}$ scale against the LNs. The mean ( \pm SEM) LN (c) and spleen (d) weights of naive ( $n=5$ per group) and immunized WT $(n=16)$ and $\operatorname{Tnf}^{\prime-}(n=14)$ mice are shown. ${ }^{A} P<0.0001$, compared with WT immunized mice.

Tnf $/$ - mice can develop severe, acute inflammatory arthritis. To evaluate the role of TNF in acute arthritis, we employed a model that develops over 1 week and is dependent on exogenous IL-1 $(17,21)$. Mild inflammation induced by a single intra-articular injection into the knee joint with mBSA is exacerbated by systemic administration of IL- $1 \beta$ on days $0-2$ and occurs in all mBSA-injected joints. Mice were sacrificed 7 days after primary injection, the time of most severe changes in the joint (21), and the knee joint sections assessed histologically. The mBSA-injected joints of $\mathrm{Tnf}^{\prime-}$ mice showed histopathological responses ranging from mild to severe. Figure 6 shows an example of the severe histopathology observed in some $\operatorname{Tnf}^{/-}$mice (Figure 6c) alongside a typical WT mouse response (Figure 6b). The response in these Tnf/- mice was similar in all respects to severely affected WT mice, with inflammatory cell infiltration, synovial thickening, and pannus-associated cartilage and bone degradation clearly evident (Figure 6d). Joint exudate cells (Figure $6 e)$ and the occasional presence of sterile microab-

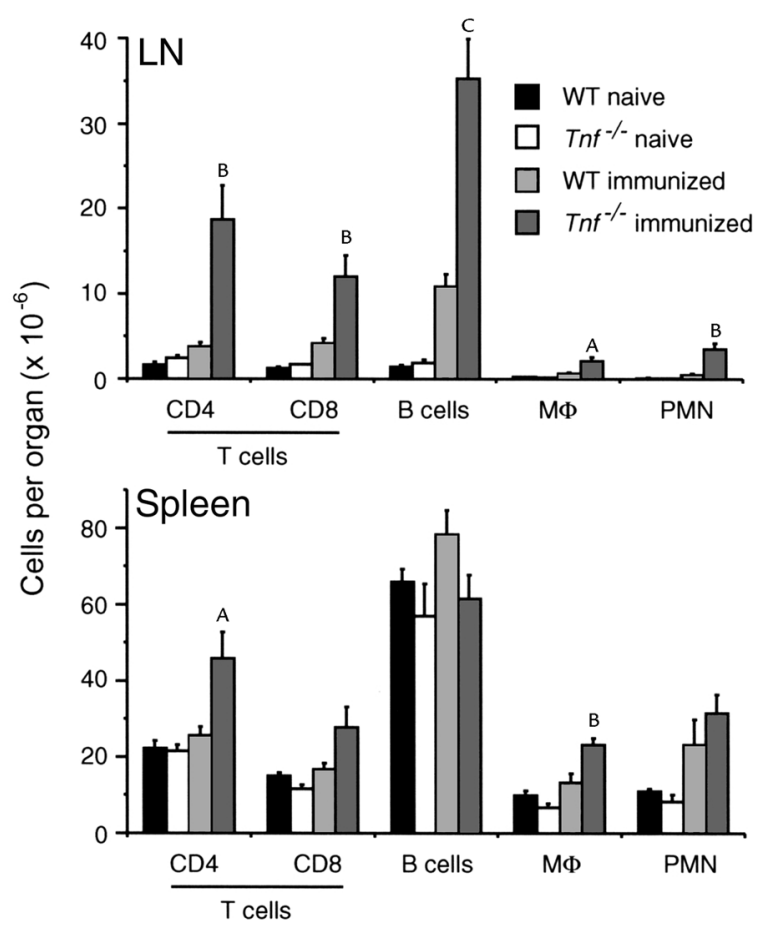


scesses in the subsynovial tissue and infrapatella fat pad (Figure 6f) were observed in both WT and Tnf ${ }^{-1}$ mice. Saline-injected contralateral control joints were normal for each genotype (Figure 6a). When knee sections were graded histologically from 0 (normal) to 5 (severe) for the features of joint space exudate, synovitis, pannus formation, cartilage, and bone loss, Tnf/mice collectively had significantly lower scores for each feature compared with WT mice (Figure $6 \mathrm{~g}$ ). The incidence of inflammatory $(9 / 22$ vs. $19 / 22$; $P<0.005)$ and destructive $(12 / 22$ vs. $20 / 22 ; P<0.01)$ arthritis were both lower in $\mathrm{Tnf}^{\prime} /$ mice compared with WT mice.

Normal inflammatory cytokine expression in synovial tissue of Tnf $/-$ mice. To determine which proinflammatory cytokines could be contributing to arthritis that develops in the absence of TNF, RNase protection analysis (RPA) was performed on RNA extracted from the synovial tissues of Tnf ${ }^{\prime-}$ and WT mice 7 days after the induction of acute inflammatory arthritis. With the exception of TNF, which was absent from the Tnf $/$ - synovium (Figure $7 \mathrm{~b}$ ), the profiles of key proinflammatory cytokines and IL-1Ra were similar for $\mathrm{Tnf}^{/-}$and WT mice (Figure 7a). Relative to the housekeeping control L32, mRNA for IL-1 $\alpha$, IL-1 $\beta$, IL-1Ra, IL-18, IL-6, IFN- $\gamma$, and MIF were detected at comparable levels for each genotype. Muscle, which served as a negative control, showed protected fragments for IL- $1 \alpha$ and MIF only.

\section{Discussion}

There is considerable evidence suggesting an important role for TNF in RA. TNF has been detected in RA synovial fluids and tissue $(22,23)$ and is a potent modulator of leukocyte, synovial cell, and chondrocyte function $(24,25)$. In animal studies, dysregulated TNF production led to chronic, inflammatory polyarthritis, which was inhibited by mAb's to TNF (26). Blockade of TNF activity with mAb's $(27,28)$ or TNF receptor/Fc

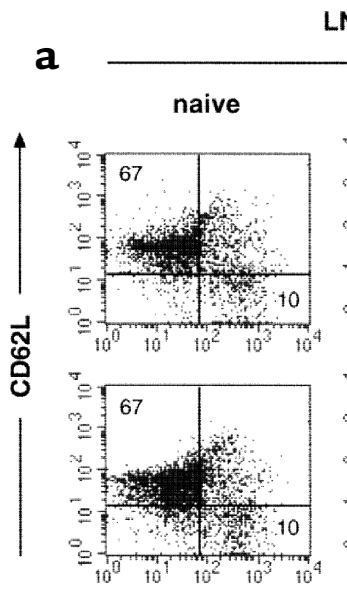

LN

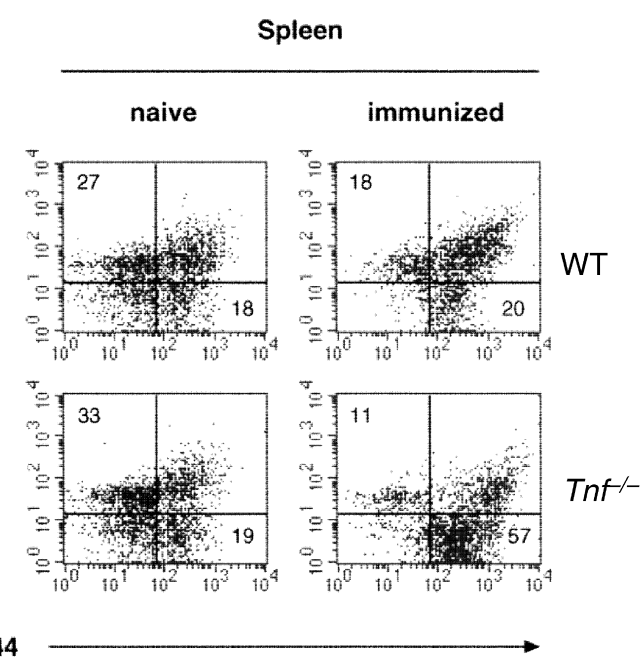

\section{CD44}
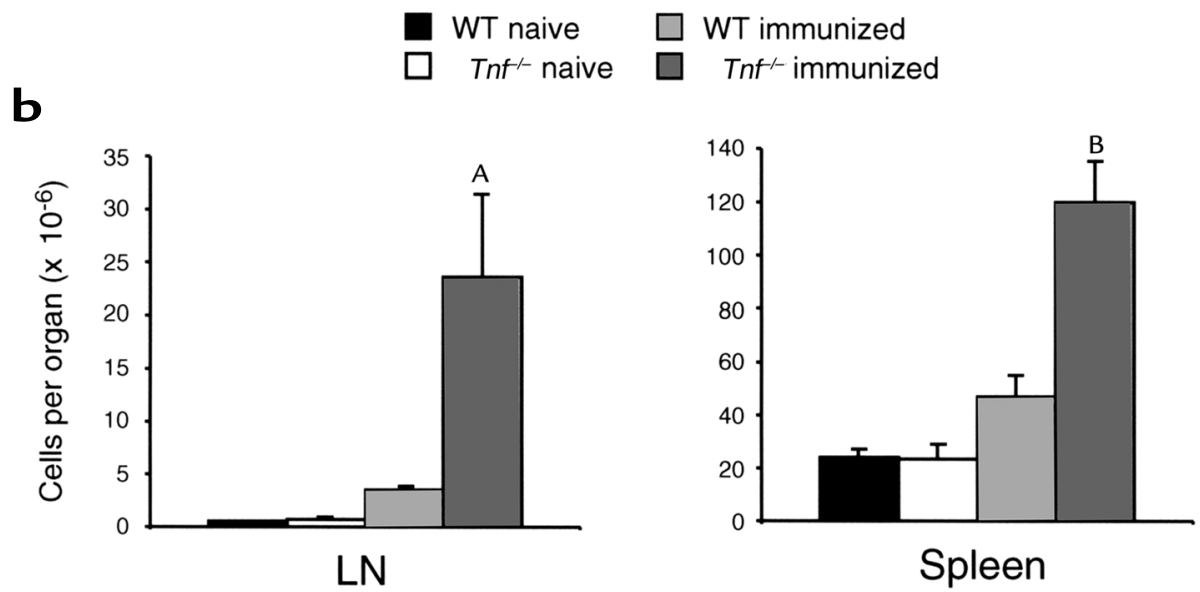

\section{Figure 5}

Increased numbers of $\mathrm{CD}^{+}$memory $\mathrm{T}$ cells in the inguinal LN and spleen of Cll-immunized $\mathrm{Tnf}^{\prime-}$ mice. $\mathrm{LN}$ and spleen cells were prepared from mice as in Figure 3 and stained for CD4, CD62L, and CD44 expression. (a) Representative FACS dot plot of CD4 ${ }^{+}$-gated cells showing staining for CD62L and CD44. Numbers in the quadrants indicate percentages of gated cells. Increased percentages of CD4 ${ }^{+}$memory $T$ cells $\left(\mathrm{CD} 62 \mathrm{~L}^{\mathrm{lo}}, \mathrm{CD}_{4}{ }^{\mathrm{hi}}\right.$ ) were observed in both the inguinal $\mathrm{LNs}$ and spleens of Cll-immunized $\mathrm{Tnf}^{\prime-}$ - mice compared with WT mice. (b) Data summarized from three such experiments showing the mean $( \pm \mathrm{SEM})$ numbers of CD4+ memory $\mathrm{T}$ cells in the inguinal $\mathrm{LN}$ and spleen of naive and Cll-immunized $\mathrm{Tnf}^{\prime-}$ and WT mice. ${ }^{\mathrm{A} P}<0.05$; ${ }^{\mathrm{B} P}<0.01$, compared with WT immunized mice. 

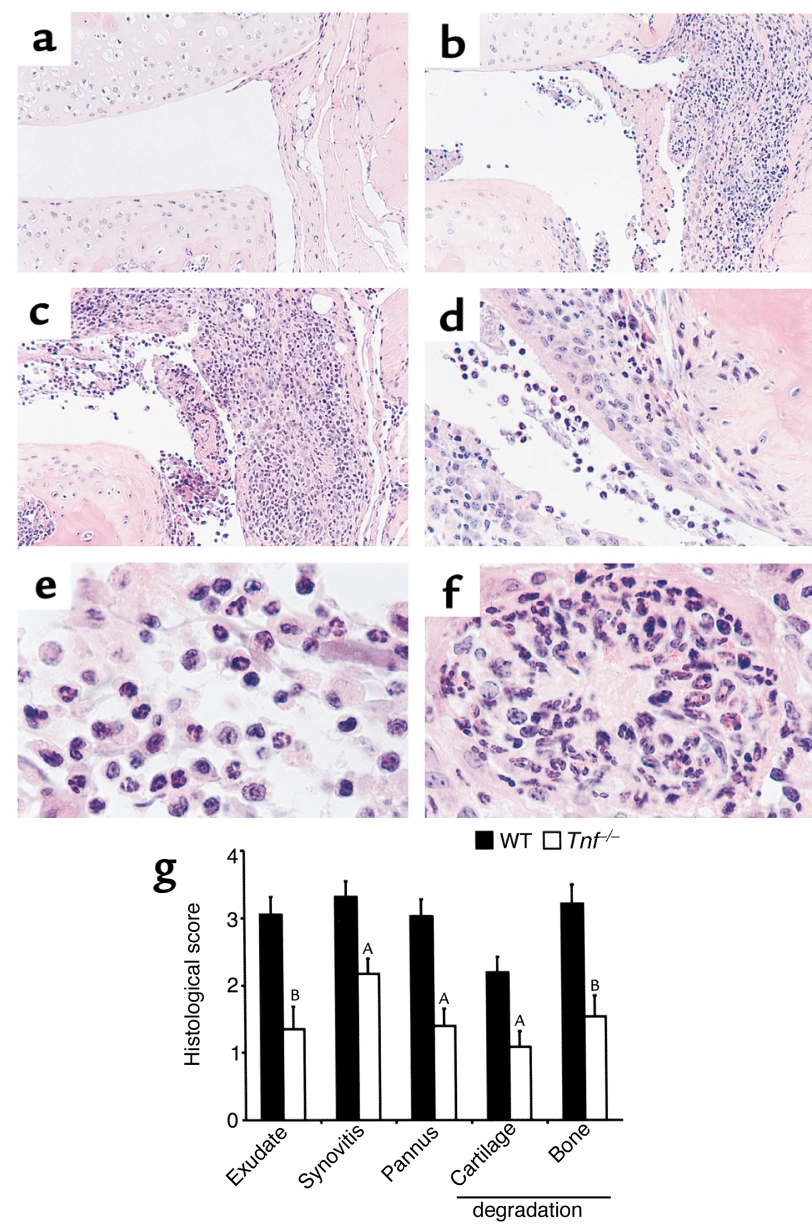

(TNFR/Fc) fusion proteins $(7,29)$ reduced the severity of CIA in mice. Consequently, TNF has been postulated as having a pivotal role in RA (3). Biological antagonists of TNF have been introduced recently for the treatment of RA and represent the first attempt to modify the course of a chronic autoimmune disease through blockade of a single cytokine. Treatment with mAb's to TNF or with soluble TNFR has produced impressive responses in some patients with RA, which has been interpreted as validation of the TNF hypothesis (30). However, not all patients with RA respond to TNF antagonists (31), and the absolute requirement for TNF in immune models of joint inflammation has never been directly examined.

In previous studies using neutralizing Ab's to TNF (27, 28 ) or soluble TNFR $(7,29)$ in the CIA model, it has not been clear whether residual disease was due to incomplete neutralization of TNF at all relevant sites. TNFR1deficient mice, backcrossed onto the DBA/1 background, showed reduced and delayed CIA compared with WT mice (7). However, since soluble TNFRs also function as physiological antagonists of TNF through competition with membrane-bound receptors for ligand, studies in mice lacking only TNFR1 but still retaining TNFR2 do not necessarily predict the effects of complete TNF deficiency on disease.

In this study, we examined Tnf/- mice in both CIA and acute destructive arthritis (mBSA/IL-1) models to deter-

\begin{abstract}
Figure 6
Histopathology of joints from $\mathrm{Tnf}^{/-}$and WT mice in a model of acute inflammatory arthritis. On day 0 , mice were injected intra-articularly into the left knee joint with mBSA $(200 \mu \mathrm{g})$ and into the right knee joint with vehicle (control). Human IL$1 \beta(250 \mathrm{ng})$ was injected subcutaneously on days 0,1 , and 2 . At day 7 , mice were sacrificed, their hind limbs removed, and the paws processed for histology as described in Methods. Frontal sections of control-injected (a) and mBSA-injected knee joints of WT (b) and $\mathrm{Tnf}^{-1}$ mice (c) are shown. (d-f) Higher magnification views of the histopathological features of arthritic Tnf ${ }^{--}$mouse joints. Hematoxylin and eosin stained sections, original magnification: $(\mathbf{a}-\mathbf{c}) \times 200,(\mathbf{d}) \times 400$, and $(\mathbf{e}$, f) $\times 1,000$. (g) Histological assessment of acute inflammatory arthritis in $\mathrm{Tnf}^{-1}$ and WT mice. Joint sections were assessed histologically for five features of inflammatory arthritis, each on a scale of 0 (normal) to 5 (severe). Results are pooled from two experiments and show the mean plus SEM $(n=22)$ values for each of the histological features. Contralateral knee joints injected with saline in lieu of $\mathrm{mBSA}$ scored 0 for all categories (not shown). ${ }^{A} P<0.005 ;{ }^{B} P<0.001$, compared with WT mice.
\end{abstract}

mine whether TNF is an absolute requirement for the development of inflammatory arthritis. Our data showed that TNF is important, but is not essential for full expression of either acute or chronic arthritis. Based on histological analyses, $\mathrm{Tnf}^{/-}$mice showed a significantly reduced degree of arthritis in each model; however, in some individuals severe disease still occurred. These mice showed disease features characteristic of those observed in severely affected WT mice. Namely, there was marked inflammatory cell infiltrate, comprising both mononuclear and polymorphonuclear cells, within the joint space and subsynovium. Extensive cartilage and bone erosions associated with fibrous-like pannus tissue were also evident. Therefore, in the absence of TNF, both the inflammatory and destructive components of disease could proceed. Arthritis in Tnf/mice might therefore parallel variable clinical responses to TNF antagonists in RA and provide valuable insights into TNF-independent mechanisms.

If TNF is the critical initiator of cytokine production in inflammatory arthritis, substitution of the next major "downstream" cytokine should compensate for the absence of TNF. The dependence of our acute model of arthritis on IL-1 also allowed us to test this hypothesis. IL-1 treatment did produce a similar profile of key inflammatory cytokines in acutely inflamed synovium of Tnf ${ }^{\prime-}$ and WT mice; however, the overall severity of arthritis in this model was in fact reduced in Tnf/- mice. These results are not consistent with a simple, dependent relationship between TNF and IL-1 and suggest that more complex interactions between TNF, IL-1, and other inflammatory mediators occur in vivo. In terms of human disease, this scenario implies greater variability in the cytokine dysregulation that occurs in RA, including the possibility that TNF may not be important in some patients.

Our studies of CIA using Tnf/- mice revealed several unexpected findings, which have potential clinical 
Table 1

$\mathrm{ClA}$ in Tnf $/-$ and WT mice ${ }^{\mathrm{A}}$

\begin{tabular}{|c|c|c|c|c|}
\hline \multicolumn{5}{|c|}{ Clinical score ${ }^{C}$} \\
\hline Group & Incidence $(\%)^{\mathrm{B}}$ & Range (median) & Mean \pm SEM & Day of onset \\
\hline WT & $12 / 28(43)$ & $1-12(4.5)$ & $4.6 \pm 1.0$ & $39.2 \pm 3.3$ \\
\hline $\operatorname{Tnf}^{\prime-}$ & $8 / 26(31)$ & $1-12(3)$ & $3.7 \pm 1.2$ & $42.8 \pm 3.9$ \\
\hline
\end{tabular}

matory arthritis, seems paradoxical. TNF regulates leukocyte trafficking through effects on chemokine production and expression of endothelial cell adhesion molecules (42-44). Although most attention has focused on the synovium, our data suggest the beneficial effect of TNF antagonism in RA may be partly due to impaired leuko-

implications. Isotype switch from IgM to IgG is part of the normal B cell response to persistent antigen. We show that TNF - whether through direct effects on B cells or indirectly through effects on LN cellular organization or CD40 ligand - plays an important role in this process. Decreased production of anti-CII IgG could account for reduced CIA because this model requires $B$ and $T$ cell responses to CII $(12,19)$. Chronic TNF blockade in patients may therefore reduce isotype switching in $B$ cells, which could increase susceptibility to infection and impair vaccination responses. In contrast, the $\mathrm{T}$ cell proliferative response to CII was preserved in $\mathrm{Tnf}^{/ /}$mice, and IFN- $\gamma$ production was actually enhanced. Lymphadenopathy and splenomegaly, associated with an increase in the number of $\mathrm{CD}^{+}$memory $\mathrm{T}$ cells at both sites and with activated B cells in inguinal LNs, was another unexpected finding in $\mathrm{Tnf}^{/-}$mice. This was only evident after immunization and was most marked several weeks after the secondary boost. Previous studies of Corynebacterium parvum infection in Tnf $^{-/}$mice showed decreased early disease, but greater infection and tissue damage subsequently (14). TNF is conventionally considered a proinflammatory cytokine, but these results imply a role for TNF in limiting inflammatory responses. Consistent with this, exogenous TNF can delay the development of murine autoimmune lupus nephritis (32) and prevent diabetes in NOD mice (33), while TNF deficiency can result in the acceleration of lupus nephritis (34) and more severe experimental autoimmune encephalomyelitis in mice (35). Similar biphasic roles have been recently discovered for other cytokines, such as IL-12 and IFN- $\gamma(36-38)$.

To our knowledge, lymphadenopathy or splenomegaly has not been a feature of patients treated with TNF antagonists, but new ANAs are relatively frequent, and increased numbers of IFN- $\gamma$ producing cells are found in the peripheral blood (39). Interestingly, treatment of RA patients with IFN- $\gamma$ was also associated with new ANAs (40). Despite a greater number of memory $\mathrm{CD}^{+} \mathrm{T}$ cells in the $\mathrm{LN}$ population and increased IFN- $\gamma$ production, we found no apparent enhancement of antigen-induced $\mathrm{T}$ cell proliferation in Tnf ${ }^{\prime-}$ mice. TNF may therefore downregulate IFN$\gamma$ production, mediate apoptosis of memory $\mathrm{T}$ cells (41) or inhibit the development of Th1 T cells. Accumulation of CII-reactive T cells, but reduced inflam-

cyte exit from the lymphoid compartment.

The introduction of TNF antagonists for the treatment of RA has been a major clinical advance and is an opportunity to gain further insight into the pathogenesis of this common disease. Preclinical data, together with some dramatic clinical responses to TNF antagonists, led to an important hypothesis regarding the regulation of cytokine production in joints by TNF. However, our data are not consistent with TNF playing an obligatory role in a cytokine cascade leading to inflammatory arthritis and suggest that more complex biological interactions occur in vivo. Most importantly, we show that TNF is dispensable for severe inflammatory joint disease, which provides a strong rationale for investigation of TNFindependent pathways in RA.

\section{Acknowledgments}

This work was supported by the Reid Charitable Trusts, the Arthritis Foundation of Australia, and the National Health and Medical Research Council of Australia. We thank J. Sedgwick (Centenary Institute, Syd-

\section{b} $\begin{array}{ccc}\text { Tnf: } & +/+ & -/- \\ \text { Tissue: } & \text { S } & \text { s }\end{array}$ a $\begin{array}{ccccc}\text { Tnf: } & +/+ & -/- & +/+ & -/- \\ \text { ssue: } & m & m & \mathrm{~S} & \mathrm{~S}\end{array}$

$=-\mathrm{E}-\mathrm{L} 32$

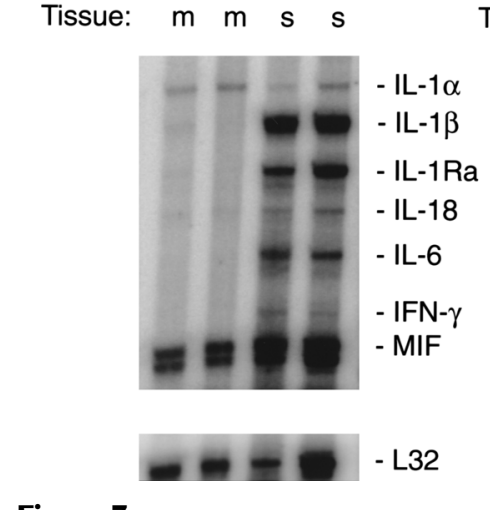

\section{Figure 7}

Cytokine mRNA expression in synovial tissue from $\mathrm{Tnf}^{-/}$and WT mice with acute inflammatory arthritis. Synovium (s) of acutely arthritic knee joints or adjacent muscle $(\mathrm{m})$ was dissected from $\mathrm{Tnf}^{-/}$ $(-/-)$ and WT (+/+) mice. Total RNA was extracted and cytokine mRNA levels assessed by RPA. (a) Synovial cytokine expression patterns were similar for $\mathrm{Tnf}^{\prime-}$ - and WT mice. Tissue specificity is shown by the lack of cytokine expression in muscle. (b) Presence of TNF mRNA in WT but not in $\mathrm{Tnf}^{-1}$ synovial extracts. Each lane represents tissue pooled from five mice with L32 being a control for RNA loading. Data are representative of two experiments performed. 
ney, Australia) and M. Smyth (Austin Research Institute, Melbourne, Australia) for the provision of Tnf/mice, and J. Melny and T. Kay for performing the ANA assay. We are grateful to $\mathrm{P}$. Egan for helpful discussion, A. Strasser for critical review of the manuscript, A. Milligan for animal care, S. Mihajlovic for histology, and D. Advani for help with figures.

1. Bazzoni, F., and Beutler, B. 1996. The tumor necrosis factor ligand and receptor families. N. Engl. J. Med. 334:1717-1725.

2. Brennan, F.M., Chantry, D., Jackson, A., Maini, R., and Feldmann, M. 1989. Inhibitory effect of TNF $\alpha$ antibodies on synovial cell interleukin1 production in rheumatoid arthritis. Lancet. 2:244-247.

3. Feldmann, M., Brennan, F.M., and Maini, R.V. 1996. Rheumatoid arthritis. Cell. 85:307-310.

4. Myers, L.K., Rosloniec, E.F., Cremer, M.A., and Kang, A.H. 1997. Collagen-induced arthritis, an animal model of autoimmunity. Life Sci. 61:1861-1878

5. Alonzi, T., et al. 1998. Interleukin 6 is required for the development of collagen-induced arthritis. J. Exp. Med. 187:461-468.

6. Manoury-Schwartz, B., et al. 1997. High susceptibility to collageninduced arthritis in mice lacking IFN- $\gamma$ receptors. J. Immunol. 158:5501-5506.

7. Mori, L., Iselin, S., De Libero, G., and Lesslauer, W. 1996. Attenuation of collagen-induced arthritis in 55-kDa TNF receptor type 1 (TNFR1)IgG1-treated and TNFR1-deficient mice. J. Immunol. 157:3178-3182.

8. Bullard, D.C., et al. 1996. Reduced susceptibility to collagen-induced arthritis in mice deficient in intercellular adhesion molecule-1. $J$. Immunol. 157:3153-3158.

9. McIntyre, K.W., et al. 1996. Reduced incidence and severity of collageninduced arthritis in interleukin-12-deficient mice. Eur. J. Immunol. 26:2933-2938.

10. Plows, D., Kontogeorgos, G., and Kollias, G. 1999. Mice lacking mature $\mathrm{T}$ and $\mathrm{B}$ lymphocytes develop arthritic lesions after immunization with type II collagen. J. Immunol. 162:1018-1023.

11. Kleinau, S., Martinsson, P., and Heyman, B. 2000. Induction and suppression of collagen-induced arthritis is dependent on distinct Fc $\gamma$ receptors. J. Exp. Med. 191:1611-1616.

12. Campbell, I.K., Hamilton, J.A., and Wicks, I.P. 2000. Collagen-induced arthritis in C57BL/6 (H-2 $)$ mice: new insights into an important disease model of rheumatoid arthritis. Eur. J. Immunol. 30:1568-1575.

13. Pasparakis, M., Alexopoulou, L., Episkopou, V., and Kollias, G. 1996. Immune and inflammatory responses in TNF $\alpha$-deficient mice: a critical requirement for $\mathrm{TNF} \alpha$ in the formation of primary $\mathrm{B}$ cell follicles, follicular dendritic cell networks and germinal centres, and in the maturation of the humoral immune response. J. Exp. Med. 184:1397-1411.

14. Marino, M.W., et al. 1997. Characterization of tumor necrosis factordeficient mice. Proc. Natl. Acad. Sci. USA. 94:8093-8098.

15. Körner, H., et al. 1997. Distinct roles for lymphotoxin- $\alpha$ and tumor necrosis factor in organogenesis and spatial organization of lymphoid tissue. Eur. J. Immunol. 27:2600-2609.

16. Campbell, I.K., Gerondakis, S., O'Donnell, K., and Wicks, I.P. 2000. Distinct roles for the NF- $\mathrm{KB} 1$ (p50) and c-Rel transcription factors in inflammatory arthritis. J. Clin. Invest. 105:1799-1806.

17. Lawlor, K.E., Campbell, I.K., O’Donnell, K., Wu, L., and Wicks, I.P. 2001 Molecular and cellular mediators of IL-1-dependent, acute inflammatory arthritis. Arthritis Rheum. 44:442-450.

18. Campbell, I.K., et al. 1998. Protection from collagen-induced arthritis in granulocyte-macrophage colony-stimulating factor-deficient mice. $J$. Immunol. 161:3639-3644.

19. Seki, N., et al. 1988. Type II collagen-induced murine arthritis. I. Induction and perpetuation of arthritis require synergy between humoral and cell-mediated immunity. J. Immunol. 140:1477-1484.

20. Sprent, J. 1997. Immunological memory. Curr. Opin. Immunol. 9:371-379.

21. Staite, N.D., et al. 1990. Induction of an acute erosive monoarticular arthritis in mice by interleukin-1 and methylated bovine serum albumin. Arthritis Rheum. 33:253-260.
22. Chu, C.Q., Feild, M., Feldmann, M., and Maini, R.N. 1991. Localization of tumor necrosis factor $\alpha$ in synovial tissues and at the cartilage-pannus junction in patients with rheumatoid arthritis. Arthritis Rheum. 34:1125-1132.

23. Saxne, T., Palladino, M.A., Jr, Heinegård, D., Talal, N., and Wollheim, F.A. 1988. Detection of tumor necrosis factor $\alpha$ but not tumor necrosis factor $\beta$ in rheumatoid arthritis synovial fluid and serum. Arthritis Rheum. 31:1041-1045.

24. Arend, W.P., and Dayer, J.-M. 1995. Inhibition of the production and effects of interleukin-1 and tumor necrosis factor $\alpha$ in rheumatoid arthritis. Arthritis Rheum. 38:151-160.

25. Feldmann, M., Brennan, F.M., and Maini, R.N. 1996. Role of cytokines in rheumatoid arthritis. Annu. Rev. Immunol. 14:397-440.

26. Keffer, J., et al. 1991. Transgenic mice expressing human tumour necrosis factor: a predictive genetic model of arthritis. EMBOJ. 10:4025-4031.

27. Williams, R.O., Feldmann, M., and Maini, R.N. 1992. Anti-tumor necrosis factor ameliorates joint disease in murine collagen-induced arthritis. Proc. Natl. Acad. Sci. USA. 89:9784-9788.

28. Joosten, L.A.B., Helsen, M.M.A., van de Loo, F.A.J., and van den Berg, W.B. 1996. Anticytokine treatment of established type II collageninduced arthritis in DBA/ 1 mice. A comparative study using anti-TNF $\alpha$, anti-IL-1 $\alpha / \beta$, and IL-1Ra. Arthritis Rheum. 39:797-809.

29. Wooley, P.H., Dutcher, J., Widmer, M.B., and Gillis, S. 1993. Influence of a recombinant human soluble tumor necrosis factor receptor $\mathrm{Fc}$ fusion protein on type II collagen-induced arthritis in mice. J. Immunol. 151:6602-6607

30. Feldmann, M., and Maini, R.N. 2001. Anti-TNF $\alpha$ therapy of rheumatoid arthritis: what have we learned? Annu. Rev. Immunol. 19:163-196.

31. Moreland, L.W., et al. 1999. Etanercept therapy in rheumatoid arthritis. A randomized, controlled trial. Ann. Intern. Med. 130:478-486.

32. Jacob, C.O., and McDevitt, H.O. 1988. Tumour necrosis factor- $\alpha$ in murine autoimmune 'lupus' nephritis. Nature. 331:356-358.

33. Jacob, C.O., Aiso, S., Michie, S.A., McDevitt, H.O., and Acha-Orbea, H. 1990. Prevention of diabetes in nonobese diabetic mice by tumor necrosis factor (TNF): similarities between TNF- $\alpha$ and interleukin 1. Proc. Natl. Acad. Sci. USA. 87:968-972.

34. Kontoyiannis, D., and Kollias, G. 2000. Accelerated autoimmunity and lupus nephritis in NZB mice with an engineered heterozygous deficiency in tumor necrosis factor. Eur. J. Immunol. 30:2038-2047.

35. Lui, J., et al. 1998. TNF is a potent anti-inflammatory cytokine in autoimmune-mediated demyelination. Nat. Med. 4:78-83.

36. Chu, C.-Q., Wittmer, S., and Dalton, D.K. 2000. Failure to suppress the expansion of the activated CD4 T cell population in interferon $\gamma$-deficient mice leads to exacerbation of experimental autoimmune encephalomyelitis. J. Exp. Med. 192:123-128.

37. Boissier, M.-C., et al. 1995. Biphasic effect of interferon- $\gamma$ in murine collagen-induced arthritis. Eur. J. Immunol. 25:1184-1190.

38. Joosten, L.A.B., Lubberts, E., Helsen, M.M.A., and van den Berg, W.B. 1997. Dual role of IL-12 in early and late stages of murine collagen type II arthritis. J. Immunol. 159:4094-4102.

39. Berg, L., Lampa, J., Rogberg, S., van Vollenhoven, R., and Klareskog, L. 2001. Increased peripheral T cell reactivity to microbial antigens and collagen type II in rheumatoid arthritis after treatment with soluble TNF $\alpha$ receptors. Ann. Rheum. Dis. 60:133-139.

40. Ioannou, Y., and Isenberg, D.A. 2000. Current evidence for the induction of autoimmune rheumatic manifestations by cytokine therapy. Arthritis Rheum. 43:1431-1442.

41. Lenardo, M., et al. 1999. Mature T lymphocyte apoptosis - immune regulation in a dynamic and unpredictable antigenic environment. Annu. Rev. Immunol. 17:221-253.

42. Sedgwick, J.D., Riminton, D.S., Cyster, J.G., and Körner, H. 2000. Tumor necrosis factor: a master-regulator of leukocyte movement. Immunol. Today. 21:110-113.

43. Paleolog, E.M., et al. 1996. Deactivation of vascular endothelium by monoclonal anti-tumor necrosis factor $\alpha$ antibody in rheumatoid arthritis. Arthritis Rheum. 39:1082-1091.

44. Tak, P.P., et al. 1996. Decrease in cellularity and expression of adhesion molecules by anti-tumor necrosis factor $\alpha$ monoclonal antibody treatment in patients with rheumatoid arthritis. Arthritis Rheum. 39:1077-1081. 